\title{
Revista 2019

\section{Encefalitis por anticuerpos contra el receptor de NMDA. Reporte de un caso y revisión de la literatura}

\author{
Edwin Forero ${ }^{\mathrm{a}}$ Hernán Castro Vargas ${ }^{\mathrm{b}}$
}

\begin{abstract}
Resumen: La encefalitis por anticuerpos contra el receptor de NMDA es un desorden grave, tratable y potencialmente reversible, caracterizado por la presencia de alteraciones en el comportamiento, convulsiones y trastornos del movimiento. La presencia de anticuerpos contra el receptor del glutamato (anti-NMDA) en plasma o líquido cefalorraquídeo es específico para el diagnóstico de la enfermedad. El reconocimiento temprano de la enfermedad es vital para el pronóstico del paciente, dado que el manejo precoz facilita la recuperación y reducción de la morbimortalidad. El tratamiento consiste en la utilización de corticoides, inmunoglobulina intravenosa o plasmaféresis como primera línea de terapia; además de otros inmunomoduladores, como ciclofosfamida o rituximab, como segunda línea. La recuperación es lenta, pero presenta mejoría en la medida en que los títulos de anticuerpos disminuyen y llega a una recuperación completa hasta en $75 \%$ de los pacientes.
\end{abstract}

Palabras clave: encefalitis; encefalitis autoinmune; inmunoglobulina; inmunomoduladores

Fecha de recepción: 27 de septiembre de 2018 Fecha de aprobación: 25 de mayo de 2019

Cómo citar: Forero E, Castro Vargas H. Encefalitis por anticuerpos contra el receptor de NMDA. Reporte de un caso y revisión de la literatura. Revista Med 27(2): 115-124. Disponible en: https://revistas.unimilitar.edu. co/index.php/rmed/article/view/4846

a Neurólogo Pediatra. Fellowship Neurofisiología con Énfasis en Epilepsia. Escuela de Medicina y Ciencias de la Salud, Universidad del Rosario, Bogotá DC, Colombia.

b Neurólogo. Fellowship Neurofisiología con Énfasis en Epilepsia. Escuela de Medicina y Ciencias de la Salud, Universidad del Rosario, Bogotá DC, Colombia. Correo electrónico: hernan.castrov@urosario.edu.co 


\title{
Anti-NMDA receptor encephalitis. Case Report and Literature Review
}

\begin{abstract}
Anti-NMDA receptor encephalitis is a severe, treatable, and potentially reversible disorder characterized by the presence of behavioral disturbances, seizures, and movement disorders. The presence of antibodies against the glutamate receptor (anti-NMDA) in plasma or cerebrospinal fluid is specific to the diagnosis of the disease. Early recognition of the disease is vital for the patient's prognosis since early management facilitates recovery and reduction of morbidity and mortality. Treatment consists of corticosteroids, intravenous immunoglobulin, or plasmapheresis as the first line of therapy, in addition to other immunomodulators, such as cyclophosphamide or rituximab, as the second line. Recovery is slow but improves as antibody titers decrease. Complete recovery occurs in up to $75 \%$ of patients.
\end{abstract}

Keywords: Encephalitis; autoimmune encephalitis; immunoglobulin; immunomodulators

\section{Encefalite causada por anticorpos contra o receptor nmda. Relatório de caso e revisão da literatura}

\begin{abstract}
Resumo: A encefalite causada por anticorpos contra o receptor NMDA é um distúrbio grave, tratável e potencialmente reversível, caracterizado pela presença de alterações comportamentais, convulsões e transtornos de movimento. A presença de anticorpos contra o receptor de glutamato (anti-NMDA) no plasma ou no líquido cefalorraquidiano é específica para o diagnóstico da doença. O reconhecimento precoce da doença é vital para o prognóstico do paciente, visto que o tratamento precoce facilita a recuperação e a redução da morbimortalidade. O tratamento consiste no uso de corticoides, imunoglobulina intravenosa ou plasmaférese como primeira linha de terapia. Além de outros imunomoduladores, como ciclofosfamida ou rituximabe, como segunda linha. A recuperação é lenta, mas apresenta melhora à medida que os títulos de anticorpos diminuem e a recuperação completa ocorre em até $75 \%$ dos pacientes.
\end{abstract}

Palavras-chave: encefalite; encefalite autoimune; imunoglobulina; imunomoduladores 


\section{Introducción}

En 2005, fue dado a conocer el caso de cuatro mujeres jóvenes que presentaban una sintomatología psiquiátrica aguda, convulsiones, pérdida de memoria, encefalopatía e hipoventilación, todo asociado con la presencia de teratoma ovárico. Se encontró que estas mujeres presentaban anticuerpos reactivos a los antígenos de superficie neuronales (1). En 2008, Dalmau y sus colaboradores describieron, en una serie de pacientes de sexo femenino, síntomas neuropsiquiátricos similares asociados a teratoma ovárico y encontraron que los anticuerpos hallados estaban dirigidos hacia los receptores de glutamato NMDA, NR1 y NR2 (2). En la actualidad, se sabe que el síndrome no está restringido a mujeres adultas con teratoma ovárico, dado que también se presenta en hombres y niños, en ausencia de patología neoplásica (3).

Este desorden se ha convertido en la principal causa de encefalitis autoinmune en niños y adolescentes: $40 \%$ de los pacientes son menores de 18 años (4). La enfermedad se presenta, en adultos y adolescentes, con un curso predecible e, incluso, se describen estadios que incluyen una fase prodrómica, caracterizada por síntomas inespecíficos como fiebre o síntomas de infección viral. Posteriormente, en el término de días a semanas, aparecen las alteraciones comportamentales y síntomas psiquiátricos, alteración del estado de conciencia, movimientos anormales y, por último, inestabilidad autonómica y respiratoria $(2,5)$. En niños, la sintomatología tiene una presentación diferente, con aparición temprana de alteraciones en el comportamiento, que incluyen rabietas de novo, agitación, agresividad y cambios frecuentes en el humor o en la personalidad. Asimismo, hay convulsiones, usualmente, de difícil manejo y trastornos del movimiento como disquinesias y movimientos coreicos y atetoides $(5,6)$. Este artículo se enfoca en la encefalitis anti-NMDAR en edad pediátrica.

\section{Información del paciente}

Paciente de 11 años, sexo femenino, que fue ingresada al servicio de urgencias por cuadro clínico de 7 días de evolución, iniciado con dos episodios de 4-5 minutos de duración de versión de la mirada, rigidez de extremidades superiores e inferiores, sin relajación de esfínteres, con recuperación posterior del tono. Durante el estudio intrahospitalario en otra institución había sido diagnosticada con epilepsia mioclónica juvenil e iniciado tratamiento con ácido valproico (250 mg cada 12 horas). Durante el manejo intrahospitalario, presentó labilidad emocional, ansiedad sobre su estado de salud y familia, por lo que la paciente recibió manejo no farmacológico por psiquiatría infantil. Después del egreso hospitalario, presentó un episodio de agitación psicomotora, coprolalia, agresión física y verbal a miembros de su familia; además de pensamientos de muerte.

La paciente no registró otros antecedentes adicionales; el padre manifestó dos meses de episodios de cefalea frontal (intensidad 8/10 en la escala visual análoga), no irradiada, tinnitus (acúfenos) y sensación de mareo asociada que se resolvía con acetaminofén.

\section{Hallazgos clínicos}

Frente al examen físico, la paciente se encontraba alerta y parcialmente orientada; presentaba llanto fácil e inmotivado, así como poca colaboración con el examinador. Aun así, no reveló alteraciones motoras y su examen neurológico se encontraba dentro de los límites normales.

Cuarenta y ocho horas después del ingreso, inició con crisis de características focales, conformando estatus epiléptico superrefractario. Por lo que fue necesario manejo en unidad de cuidados intensivos (UCI), con IOT, manejo con gastrostomía, traqueostomía, con múltiples anticonvulsivantes y anestésicos.

Gracias a una resonancia magnética nuclear cerebral, se evidenciaron signos de hipertensión endocraneana, con alteración en la intensidad de la señal de hipocampo bilateral, así como cuerpo de hipocampo derecho que se comporta hiperintenso en T2 y FLAIR (discreto, realce tipo paquimeningitis bilateral, Figura 1). El video-electroencefalograma reportó hallazgo anormal, compatible con lesión estructural izquierda y actividad epileptiforme en la presencia de crisis clínica. Se realizó estudio no reactivo para anticuerpos contra citomegalovirus y virus de Epstein Barr, Herpes virus tipo I y II no detectados en lcr, complemento C3 1.56, C4 0.18. Anticuerpos ADN negativos, ENAS negativo, cardiolipinas IgG e IgM negativas, anticoagulante lúpico 
positivo, radiol,49, IgA e IgG dentro de límites normales; estudio de p-anca y c-anca negativos; y estudio para anticuerpos anti-NMDA en suero y líquido cefalorraquídeo (LCR) positivos.

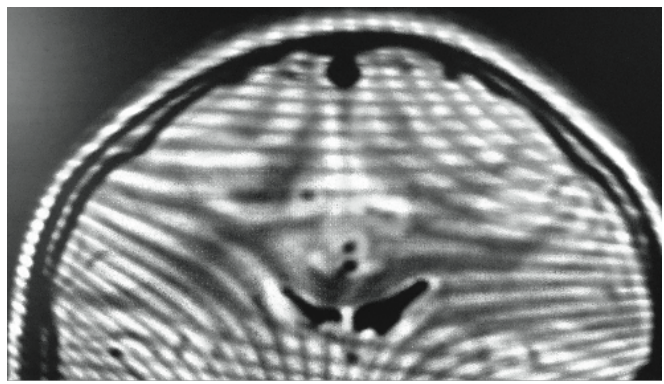

Figura 1. RNM cortes coronales y axiales con hiperintensidad en FLAIR en hipocampo bilateral con predominio derecho.

En video-electroencefalograma, se reportó asimismo un hallazgo anormal por encefalopatía moderada, en paciente en uso de medicación sedante. Así, comparando con estudio previo se evidenció disminución del grado de epileptogénesis.

\section{En cariotipo sin alteración cromosómica}

Durante su estancia en la UCI, la paciente recibió tratamiento médico con plasmaféresis y corticoides en bolo, durante cinco días. Al permanecer encefalopática, se inició manejo con inmunoglobulina asociada a ciclofosfamida. Posteriormente, una vez descartado un foco infeccioso, se inició manejo con rituximab, con lo cual la paciente mostró signos de mejoría.

En la actualidad, la paciente se encuentra en manejo con monoterapia de levetiracetam y ha presentado una adecuada evolución, sin presencia de crisis desde hace más de tres años, con evidencia de un déficit cognitivo leve. Sin embargo, se encuentra recibiendo educación regular con apoyo escolar. En resonancia nuclear magnética de cerebro, se ha evidenciado una leve acentuación de los surcos corticales de ambos hemisferios cerebrales, mayor de lo esperado para su edad (Figura 2) y trazado electroencefalográfico normal para la edad.

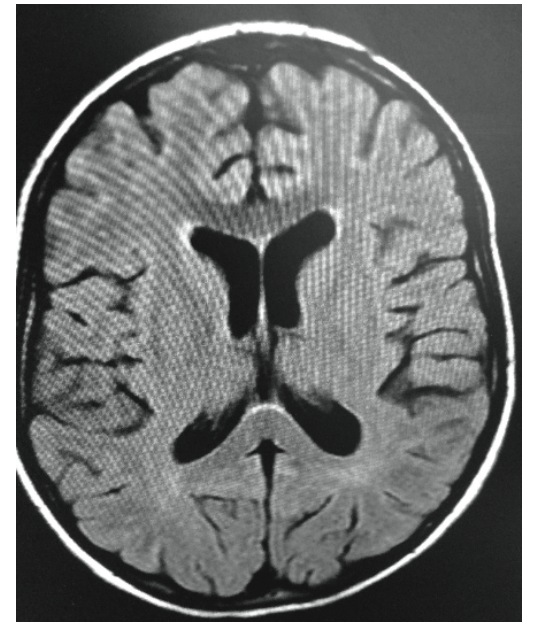

Figura 2. RNM cerebral en corte axial con evidencia de acentuación de surcos corticales mayores que los esperado para la edad.

\section{Revisión de la literatura}

\section{Definición de encefalitis por anticuerpos contra el receptor de NMDA}

La encefalitis por anticuerpos contra el receptor de N-metil-D-aspartato (NMDA) es una condición autoinmune mediada por anticuerpos antineuronales, caracterizada por presentar manifestaciones graves de la esfera mental, predominantemente, como alteraciones de la conducta, del afecto, estados de catatonia y psicosis, además de trastornos del movimiento y convulsiones (7-9).

\section{Epidemiologia}

La encefalitis anti-NMDAR una de las principales causas de encefalitis en la población pediátrica (8) y es más frecuente en pacientes jóvenes y en mujeres (9). En un estudio en Reino Unido, se determinó que $4,0 \%$ de las encefalitis, tanto de adultos como niños, correspondían a una encefalitis anti-NMDAR, por lo que es la causa inmune más común la encefalomielitis aguda diseminada [ADEM] $(5,10)$.

En una serie de casos analizados en 2009, que incluyó niños y adultos, se encontró que $40 \%$ de los casos correspondían a pacientes menores de 18 años y un niño de 23 meses de edad fue el paciente más joven. En la serie de Dalmau y sus 
colaboradores se encontró que, de los 100 pacientes revisados, 22 eran niños, los cuales tenían un tumor diagnosticado en el $55 \%$ de los casos $(2,3,9)$.

En el California Encephalitis Project (CEP), la mitad de los niños con encefalitis idiopática y con presencia de sintomatología psiquiátrica presentaba anticuerpos anti-NMDAR, con frecuencia cuatro veces mayor que las encefalitis causadas por Herpes simple, Varicela zóster y virus del Nilo occidental $(9,11)$.

Existe una importante asociación entre la presencia de tumores y el desarrollo de encefalitis anti-NMDAR. En mujeres, la frecuencia entre teratoma ovárico y encefalitis es aproximadamente $62 \%$, mientras que, en hombres, solo el $22 \%$ de los pacientes tiene tumores asociados (teratoma testicular, tumor pulmonar de células pequeñas). En mujeres adolescentes menores de 18 y 14 años, se encontró asociación con tumores en $31 \%$ y $9 \%$ de los casos, respectivamente $(3,12)$.

\section{Fisiopatología}

Los receptores NMDA son complejos tetraméricos compuestos por dos subunidades NR1, con unión de glicina y NR2, con unión de glutamato. Están localizados en la membrana postsináptica, funcionan como canales catiónicos activados por ligando y tienen una gran importancia en la plasticidad y transmisión sináptica (Figura 3). Los autoanticuerpos en plasma y líquido cefalorraquídeo se ajustan a un epítope localizado en el dominio extracelular de las subunidades NR1 (13).

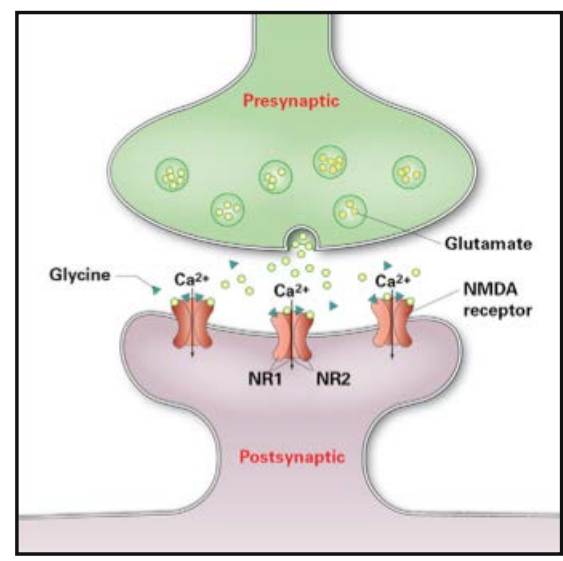

Nota: los receptores de glutamato compuestos de las unidades NR1 y NR2 se encuentran en la membrana postsináptica.

Figura 3. Receptores de glutamato tipo NMDA. Fuente: Wandinger et al.J Neuroimmunol. 2011; 231(1-2):86-91. (13).
En la fase aguda, la producción intratecal de anticuerpos anti-NMDAR induce la internalización del receptor, mientras la disminución en la expresión del receptor en la superficie neuronal produce hipoactividad neuronal. En el estadio crónico, una vez disminuyen los niveles de autoanticuerpos producidos por las células plasmáticas, empieza a restaurarse la barrera hematoencefálica, lo que disminuye los niveles de anticuerpos en el LCR y, así, se expresa nuevamente el receptor y se reestablecen las funciones neuronales (14).

\section{Manifestaciones clínicas}

La encefalitis anti-NMDAR se presenta como un síndrome con diferentes estadios que van desde un cuadro prodrómico inespecífico que puede incluir cefalea o fiebre, que evoluciona después de un periodo de la presentación de la sintomatología hasta la recuperación. El fenotipo en adultos y en niños es similar, a excepción de la disfunción autonómica y la hipoventilación, que son más frecuentes en adultos $(3,9,15)$.

Síntomas prodrómicos: los principales síntomas en el momento de presentación de la enfermedad incluyen fiebre, cefalea, rinitis, vómito y diarrea, los cuales se observan en $48-86 \%$ de los casos, hasta dos semanas antes del ingreso al hospital $(3,9)$. Los pacientes desarrollan, al menos, tres de los siguientes síntomas hasta un mes después del inicio de la enfermedad: psiquiátricos, alteraciones del lenguaje, alteraciones de memoria, convulsiones, movimientos anormales, alteración del estado de conciencia, inestabilidad autonómica e hipoventilación [Figura 4] (2).

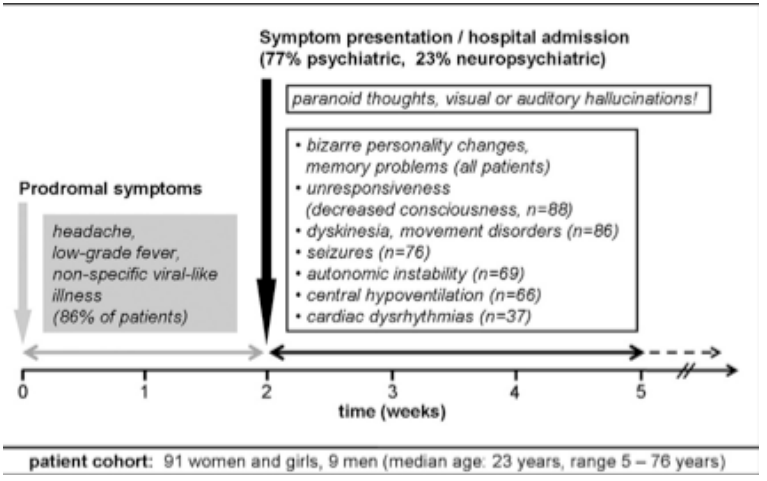

Figura 4. Características clínicas de los pacientes con encefalitis anti-NMDAR.

Fuente: Dalmau et al. Lancet Neurol. 2008; 7(12):1091-8. (2). 
Síntomas psiquiátricos: estos síntomas, que involucran la esfera mental, incluyen ansiedad, paranoia, miedo, psicosis, manía e insomnio. Las alteraciones en el comportamiento incluyen la aparición de rabietas, irritabilidad e hiperactividad y en ocasiones se observa agresividad, episodios de agitación, psicosis e incluso hipersexualidad. También han sido reportados síntomas psicóticos, manía e incluso catatonia en adolescentes con encefalitis anti-NMDAR. Con frecuencia, la sintomatología psiquiátrica es manejada, al comienzo, como un síndrome neuroléptico maligno, por la presencia de rigidez, alteración autonómica y elevación de las enzimas musculares; y se diferencia por no tener antecedentes del uso de neurolépti$\cos (12,16)$. Se ha descrito, además, la presencia de insomnio, conducta aberrante, alucinaciones, pensamiento de contenido paranoide; el lenguaje también se puede ver comprometido, aunque inicialmente pueden evidenciarse episodios de taquilalia, los cuales presentan una reducción progresiva que puede llegar a manifestarse con mutismo (9).

Síntomas neurológicos: los principales síntomas neurológicos incluyen trastornos del movimiento, convulsiones y alteraciones cognitivas. La inestabilidad autonómica y los trastornos del sueño son más frecuentes en adultos y ocurren con muy poca frecuencia en niños. En cuanto a los trastornos del movimiento, han sido reportados en cerca del $85 \%$ de los casos de niños con la enfermedad y se han encontrado disquinesias orales, coreo atetosis y distonías entre los más frecuentes, seguidos por opistótonos y rigidez. Se han descrito pocos casos de mujeres con encefalitis anti-NMDAR con presencia de opsoclono-mioclono $(9,17)$.

La presencia de disautonomías es síntoma infrecuente en niños y de escasa gravedad, como las observadas en adultos. Otros síntomas como hipertemia, hipersalivación e incontinencia urinaria son reportados con frecuencia en pacientes con encefalitis anti-NMDAR. La presencia de convulsiones tanto focales como generalizadas ha sido descrita hasta en $77 \%$ de los casos y puede presentarse desde las etapas iniciales de la enfermedad (9). El estatus epiléptico no es infrecuente en estos pacientes y ha llegado a considerarse el cuadro clínico, inicialmente, como un síndrome de Rasmussen (15).

\section{Diagnóstico}

Neuroimágenes: sigue siendo, después de las características clínicas, la herramienta de mayor utilidad en el diagnóstico de la encefalitis anti-NMDAR, a pesar de su baja especificidad. Las imágenes por tomografía axial computarizada (TAC) son de poca utilidad en el estudio de la patología debido a su poca sensibilidad. Por otro lado, la RNM cerebral puede ser normal en $50 \%$ de los casos y en el restante $50 \%$ se observan imágenes no específicas de hiperintensidades en hipocampos, corteza frontal basal, ganglios basales, tallo cerebral y cordón espinal en las secuencias T2 y FLAIR. Estos cambios pueden ser transitorios $y$, usualmente, requieren la utilización de medios de contraste para su detección. Las imágenes por espectroscopia por resonancia y las neuroimágenes funcionales (PET, SPECT) no muestran anormalidades características de la enfermedad. Es importante mencionar que existe escasa información al respecto $(9,15,18)$.

Electroencefalograma: se encuentra anormal en la mayoría de los pacientes y presenta lentificación difusa y desorganización de los ritmos de fondo. Es posible encontrar actividad epileptiforme e incluso crisis durante el episodio agudo. La presencia de actividad delta y theta continuas es más frecuente en los estadios subagudos y crónicos $(9,15)$.

Liquido cefalorraquídeo: el diagnóstico definitivo de la encefalitis anti-NMDAR está definido por la obtención y confirmación de la presencia de anticuerpos en suero o LCR, contra la subunidad NR1 del receptor NMDA. Se ha encontrado que los pacientes con teratoma poseen títulos de anticuerpos en LCR más altos que los pacientes sin teratoma. En el examen citoquímico, es frecuente encontrar un aumento de los glóbulos blancos, que llegan a niveles de 200 células $/ \mathrm{mm}^{3}$, con pleocitosis hasta en el $87 \%$ de los pacientes pediátricos. Las proteínas se encuentran dentro de los valores considerados normales o ligeramente aumentados; y también hay presencia de bandas oligoclonales, las cuales se encuentran positivas hasta en el $60 \%$ de los casos $(3,5)$.

Diagnóstico diferencial: teniendo en cuenta las manifestaciones clínicas mencionadas, debe establecerse un diagnóstico diferencial con trastornos psiquiátricos primarios, como los episodios 
psicóticos agudos, con cuadros de encefalitis de causa viral, intoxicación por medicamentos psicotrópicos y síndrome neuroléptico maligno (9).

\section{Tratamiento y pronóstico}

El manejo de la encefalitis por anticuerpos anti-NMDA está enfocado en la detección del teratoma y su posterior remoción y en el manejo con inmunoterapia. Se ha demostrado que su inicio en periodos tempranos contribuye a una recuperación más rápida y a una reducción en la morbilidad $(9,15)$.

En la actualidad la inmunoterapia incluye el manejo con corticoides, plasmaféresis, inmunoglobulina intravenosa y anticuerpos monoclonales (rituximab). La mayoría de los pacientes requiere hospitalizaciones prolongadas y monitoreo en UCI durante el manejo agudo $(15,19)$. Se recomienda el inicio precoz de pulsos intravenosos de corticoide asociado a IgG intravenosa durante cinco días, o plasmaféresis asociada a resección del tumor, en caso de presentarse (9).

La ciclofosfamida y el rituximab se consideran parte del esquema de segunda línea. Su uso solo o en combinación ha arrojado buenos resultados en el manejo de encefalitis en adultos. Sin embargo, en niños se prefiere evitar el uso de ciclofosfamida debido a los potenciales efectos adversos (malignidad, infertilidad, falla gonadal prematura) y solo se reserva para el manejo en niños que no han respondido a los otros manejos (6).

Por otro lado, dado que el manejo falla aproximadamente en un 30-40\% de los pacientes y con los crecientes reportes de efectividad del rituximab y su efecto benéfico en las tasas de recurrencia, este medicamento de segunda línea se ha venido usando en combinación con los corticoides e inmunoglobulina en la terapia de primera línea $(9,20)$.

El pronóstico depende del inicio temprano del manejo con la terapia inmunomoduladora y la remoción del tumor, en caso de que curse como un síndrome paraneoplásico. Sin embargo, a pesar de la gravedad de la sintomatología y las hospitalizaciones prolongadas, el pronóstico es, con frecuencia, positivo, ya que se estabiliza la sintomatología en el término de semanas o meses y se logra una recuperación completa hasta en el $80 \%$ de los casos (15).
Los síntomas que mejoran primero son la inestabilidad autonómica, las disquinesias, el estado de conciencia y las convulsiones. La presencia de síntomas residuales, como disfunción frontal y límbica, y la presencia de impulsividad, desinhibición y déficits de memoria, así como un deterioro en las funciones ejecutivas, no son infrecuentes (13).

\section{Referencias}

1. Vitaliani R, Mason W, Ances B, Zwerdling T, Jian Z, Dalmau J. Paraneoplastic encephalitis, psychiatric symptoms, and hypoventilation in ovarian teratoma. Ann Neurol. 2005; 58(4):594-604.

2. Dalmau J, Gleichman AJ, Hughes EG, Rossi JE, Peng $\mathrm{X}$, Dessain SK et al. Anti-NMDA-receptor encephalitis: Case series and analysis of the effects of antibodies. Lancet Neurol. 2008; 7(12):1091-8.

3. Florance NR, Davis RL, Lam C, Szperka C, Zhou L, Ahmad $S$ et al. Anti-N-methyl-D-aspartate receptor (NMDAR) encephalitis in children and adolescents. Ann Neurol. 2009; 66(1):11-8.

4. Dalmau J, Lancaster E, Martínez-Hernández E, Rosenfeld M. Balice-Gordon R. Clinical experience and laboratory investigations in patients with anti-NMDAR encephalitis. Lancet Neurol. 2011; 10(1):63-74.

5. Armangue T. Petit-Pedrol, Mar. Dalmau J. Autoimmune encephalitis in children. J Child Neurol. 2012; 27(11):1460-9.

6. Glaser C, Iizuka T, Honig LS, Aguilar E, Gresa N, Ryan $\mathrm{N}$ et al. Treatment and prognostic factors for long-term outcome in patients with anti-N-Methyl-D-Aspartate (NMDA) receptor encephalitis: a cohort study. Lancet Neurol. 2014; 12(2):157-65.

7. Warren N, Grote V, Gorman CO, Siskind D. Electroconvulsive therapy for anti-N-methyl-D-aspartate (NMDA) receptor encephalitis: A systematic review of cases. Brain Stimul. 2018; 12(2):329-34. Doi: https:// doi.org/10.1016/j.brs.2018.11.016

8. Khundakji Y, Masri A, Khuri N. Anti-NMDA receptor encephalitis in a toddler: A diagnostic challenge. Int J Pediatr Adolesc Med. 2018; 5(2):75-7. Doi: https://doi. org/10.1016/j.ijpam.2018.03.001

9. Guasp M, Dalmau J. Encefalitis por anticuerpos contra el receptor de NMDA. Med Clin (Barc). 2018; 151(2):719. Doi: https://doi.org/10.1016/j.medcli.2017.10.015

10. Granerod J, Ambrose HE, Davies NW, Clewley JP, Walsh AL, Morgan D et al. Causes of encephalitis and differences in their clinical presentations in England: A multicentre, population-based prospective study. 
Lancet Infect Dis. 2010; 10(12):835-44. Doi: http://dx. doi.org/10.1016/S1473-3099(10)70222-X

11. Gable MS, Sheriff H, Dalmau J, Tilley DH, Glaser CA. The frequency of autoimmune N-methyl-D-aspartate receptor encephalitis surpasses that of individual viral etiologies in young individuals enrolled in the California Encephalitis Project. Clin Infect Dis. 2012; 54:899904.

12. Florance-Ryan N, Dalmau J. Update on anti-N-methyl-D-aspartate receptor encephalitis in children and adolescents. Curr Opin Pediatr. 2010; 22:739-44.

13. Wandinger K, Saschenbrecker S, Stoecker W, Dalmau J. Anti-NMDA-receptor encephalitis: A severe, multistage, treatable disorder presenting with psychosis. J Neuroimmunol. 2011; 231(1-2):86-91. Doi: http://dx. doi.org/10.1016/j.jneuroim.2010.09.012

14. Miya K, Takahashi Y, Mori H. Anti-NMDAR autoimmune encephalitis. Brain Dev. 2014; 36(8):645-52. Doi: http://dx.doi.org/10.1016/j.braindev.2013.10.005

15. Jones KC, Benseler SM. Anti-NMDA receptor encephalitis. Neuroimaging Clin N Am. 2013; 23(2):30920. Doi: http://dx.doi.org/10.1016/j.nic.2012.12.009
16. Consoli A, Ronen K, An-gourfinkel I, Barbeau M, Marra D, Costedoat $\mathrm{N}$ et al. Malignant catatonia due to anti-NMDA-receptor encephalitis in a 17 -year-old girl: Case report. Child Adolescent Psychiatry Ment Heal. 2011; 5(15):2-7.

17. Kurian M, Lalive P, Dalmau J, Horvath J. Opsoclonus-myoclonus syndrome in anti-N-methyl-D-aspartate receptor encephalitis. Arch Neurol. 2010; 67(1):1-5.

18. Agundez M, Mendibe M, Carlos J, Ciordia R, Saiz A, Zarranz JJ. Abnormal multifocal cerebral blood flow on Tc-99m HMPAO SPECT in a patient with anti-NMDA-receptor encephalitis. J Neurol. 2010; 257:1568-9.

19. Peery HE, Day GS, Dunn S, Fritzler MJ, Prüss H, Souza $\mathrm{C}$ et al. Autoimmunity reviews anti-NMDA receptor encephalitis. The disorder, the diagnosis and the immunobiology. Autoimmun Rev. 2012;11(12):863-72. Doi: http://dx.doi.org/10.1016/j.autrev.2012.03.001

20. Wong-Kisiel LC, Ji T, Renaud DL, Kotagal S, Patterson MC, Dalmau J et al. Response to immunotherapy in a 20-month-old boy with anti-NMDA receptor encephalitis. Neurology. 2010; 74:1550-1. 

